\title{
Emerging Pharmacological Treatment in Nonalcoholic Steatohepatitis
}

\author{
Jörn M. Schattenberg \\ Metabolic Liver Research Program, First Department of Medicine, University Medical Center Mainz, Mainz, Germany
}

\section{Keywords}

Nonalcoholic fatty liver disease · Clinical trials .

Nonalcoholic steatohepatitis - Metabolic liver disease ·

End-stage liver disease · Pharmacotherapy

\begin{abstract}
Background: The increasing prevalence of nonalcoholic fatty liver disease has led to a strong demand for an optimal therapeutic approach. At present, guidelines recommend lifestyle changes, but it has become apparent that pharmacotherapy will be required in patients with advanced disease to prevent the progression to end-stage liver disease and potentially improve extrahepatic outcomes. Summary: This review discusses current pharmacological approaches focusing on substances studied in pivotal trials and selected phase 2 trials in patients with nonalcoholic steatohepatitis (NASH) and fibrosis. Key Message: Currently, several compounds are subjected to clinical testing to explore predominantly anti-inflammatory, anti-fibrotic, and metabolic treatment for NASH. With current response rates around 20\%, the combination of several drugs targeting more than one pathway could lead to increased treatment success in the future.
\end{abstract}

\section{Introduction}

Nonalcoholic fatty liver disease (NAFLD) is a major cause of liver disease globally. Nonalcoholic fatty liver and nonalcoholic steatohepatitis (NASH) are two distinct histologically defined subforms which require different prognostic and therapeutic approaches. NASH is considered an inflammatory form with hepatocyte injury that can progress to liver cirrhosis and hepatocellular carcinoma (HCC). In particular, advanced fibrosis resulting from NASH is linked to increased overall mortality [1] and can be prevalent in high-risk groups such as patients with type 2 diabetes [2]. Although current guidelines recommend a lifestyle aiming at weight loss to be achieved through increasing physical activity and dietary changes, not all patients respond to these measures and pharmacotherapy is eagerly awaited for subgroups, in particular patients with highly active or advanced disease. As a matter of fact, despite the broad consensus that lifestyle changes are beneficial to obese patients with metabolic diseases, only a minority of patients can be effectively treated with lifestyle modifications. In a large, prospective study assessing lifestyle interventions on cardiovascular outcomes, the Look AHEAD study, intensive lifestyle 
Table 1. Drugs currently explored in phase 3 clinical trials

\begin{tabular}{|c|c|c|c|c|c|}
\hline Drug & Elafibranor & Cenicriviroc & Obeticholic acid & Aramchol & $\begin{array}{l}\text { Resmetirom } \\
\text { (MGL-3196) }\end{array}$ \\
\hline $\mathrm{MoA}$ & $\mathrm{PPAR} \alpha / \delta$ agonist & $\begin{array}{l}\text { CCR2/CCR5 } \\
\text { antagonist }\end{array}$ & FXR agonist & $\begin{array}{l}\text { SCD-1 } \\
\text { modulator }\end{array}$ & $\begin{array}{l}\text { Thyroid- } \beta \\
\text { agonist }\end{array}$ \\
\hline Study name & $\begin{array}{l}\text { RESOLVE-IT } \\
\text { (NCT02704403; } \\
\text { Genfit, Loos, France) }\end{array}$ & $\begin{array}{l}\text { AURORA } \\
\text { (NCT03028740; } \\
\text { Allergan, Dublin, } \\
\text { Ireland) }\end{array}$ & $\begin{array}{l}\text { REGENERATE } \\
\text { (NCT02548351; } \\
\text { Intercept } \\
\text { Pharmaceuticals, New } \\
\text { York, NY, USA) }\end{array}$ & $\begin{array}{l}\text { ARMOR } \\
\text { Galmed } \\
\text { Pharmaceuticals, } \\
\text { Tel Aviv, Israel) }\end{array}$ & $\begin{array}{l}\text { MAESTRO-NASH } \\
\text { (NCT03900429; } \\
\text { Madrigal } \\
\text { Pharmaceuticals, } \\
\text { West Conshohocken, } \\
\text { PA, USA) }\end{array}$ \\
\hline Study population, $n$ & 2,000 & 2,000 & 2,480 & 2,000 & 2,000 \\
\hline $\begin{array}{l}\text { Phase } 2 \text { results } \\
\qquad \text { Follow-up, weeks }\end{array}$ & $\begin{array}{l}\text { GOLDEN } \\
(n=276) \\
52\end{array}$ & $\begin{array}{l}\text { CENTAUR } \\
(n=289) \\
54\end{array}$ & $\begin{array}{l}\text { FLINT } \\
(n=273) \\
72\end{array}$ & $\begin{array}{l}\text { ARREST } \\
(n=247) \\
52\end{array}$ & $\begin{array}{l}\text { MGL-3196 } \\
(n=125) \\
12\end{array}$ \\
\hline $\begin{array}{l}\text { Follow-up, weeks } \\
\text { Improvement }\end{array}$ & 52 & 54 & 72 & 52 & 12 \\
\hline NAS & + (post hoc) & - & + & - & $-{ }^{1}$ \\
\hline Fibrosis & (+) Responder (post hoc) & $\begin{array}{l}+(20 \text { vs. } \\
10 \% \text { placebo })\end{array}$ & + & $\begin{array}{l}+(29.5 \text { vs. } \\
17.5 \% \text { placebo })\end{array}$ & \\
\hline Safety & $\begin{array}{l}\text { Reversible increase } \\
\text { in creatinine) }\end{array}$ & - & $\begin{array}{l}\text { LDL cholesterol } \\
\text { increase, pruritus } \\
\text { in } 50 \%\end{array}$ & - & $\begin{array}{l}\text { Mild diarrhea and } \\
\text { GI side effects }\end{array}$ \\
\hline
\end{tabular}

${ }^{1}$ Histology in substudy only; here, effect on NASH and fibrosis.

changes did not improve outcome at 8 years [3]. This highlights the urgent need to establish effective and safe pharmacological treatment for NAFLD patients. At present, several drugs are in late-stage clinical development for fibrotic NASH and will be summarized in this review (Table1). Given that NASH is a multifactorial disease, a large number of biological pathways are involved and therefore explored to reduce disease activity.

\section{Current Recommendations: Effect of Lifestyle Changes on NASH}

At present, lifestyle changes constitute the first-line management of NASH aiming at weight loss to be achieved through increasing energy expenditure and reducing caloric intake. In one prospective trial, a weight loss of 7-10\% was required to reduce hepatic inflammation and fibrosis [4]. In this trial, only $50 \%$ of patients achieved weight loss $>5 \%$, reflecting the well-known problem of reaching and maintaining sufficient weight loss [3]. The effect of a formula diet on hepatic steatosis has recently been tested in patients with morbid obesity in Germany [5]. The effects of caloric restriction to $800 \mathrm{kcal} /$ day or 12 weeks and the recommendation for a protein-rich, but overall balanced diet was investigated during a 52-week follow-up. Here, weight loss $>10 \%$ was associated with a significant decline in ALT and an improvement in hepatic steatosis. Even patients with normal BMI showed benefits from weight loss in a study that was performed in Asia [6]. The most commonly recommended lifestyle is the so-called Mediterranean diet, which is rich in monounsaturated fats, fruits, vegetables, nuts, and low in processed foods, as well as red meat, which has been studied extensively in cardiovascular disease but also in obesity [7]. In a randomized, controlled trial comparing low-fat versus a Mediterranean/low-carbohydrate diet over 18 months in patients with NAFLD, hepatic fat was more effectively reduced in the Mediterranean diet group [8]. Although physical activity is improving metabolic control and is recommended in patients with NAFLD, most patients fail to adhere to exercise recommendations. Thus, supported exercise programs are needed as they give a guiding framework based on ability and exhaustion. Even a short, web-based, individualized exercise program of only 8 weeks improved markers of steatosis, inflammation, and fibrosis [9]. While bariatric surgery that is indicated in patients with morbid obesity and complications of type 2 diabetes does lead to the resolution of steatohepatitis in the sequel of the weight loss [10], it is currently not recommended as first-line therapy in patients with NAFLD.

\section{Pharmacological Treatment}

\section{Nuclear Receptor-Targeting Drugs}

At present, several drugs targeting nuclear receptors are in development, including agonists for the farnesoid 
$\mathrm{X}$ receptor (FXR) and the peroxisome proliferator-activated receptors (PPARs). Pioglitazone, a PPAR $\gamma$ agonist, has previously been shown to improve steatohepatitis in patients without diabetes when combined with vitamin $\mathrm{E}$ [11]. This was recapitulated in more recent trials in patients with diabetes [12]. However, pioglitazone is not widely used due to safety concerns and limited availability in some EU countries [13].

\section{Obeticholic Acid}

The most advanced drug in clinical development is the FXR agonist obeticholic acid (Ocaliva; OCA; INT-747). OCA is a semisynthetic analogue of the bile acid chenodeoxycholic acid and a potent FXR agonist. Its efficacy and safety were shown in FLINT, a phase 2 trial. This large multicenter phase $2 \mathrm{~b}$ trial included 283 patients and was terminated early when the primary endpoint (a $>2$-point improvement in the NAFLD activity score with no worsening of liver fibrosis) was met in $45 \%$ of the treatment group and $21 \%$ of the placebo group [14]. Adverse effects included pruritus in $23 \%$ of the OCA-treated patients versus $6 \%$ in the placebo group, as well as elevated levels of low-density lipoprotein (LDL) [14]. First results ever reported come from an interim analysis of the REGENERATE study (ClinicalTrials.gov identifier, ID: NCT02548351), a phase 3 trial including NASH patients. This trial achieved its primary endpoint and demonstrated statistically significant improvement in liver fibrosis without worsening of NASH at 18 months at a dose of 25 $\mathrm{mg} /$ daily [15]. Safety concerns occurred with pruritus being reported in up to $50 \%$ of the $25-\mathrm{mg}$ treatment group, and LDL levels were increased. However, these results have to be put into perspective, as most patients with NASH exhibited a heightened cardiovascular risk event, and when modeling the safety profile observed in the REGENERATE trials, only $8 \%$ of all patients showed changes with respect to the risk of a cardiovascular event [16].

\section{Elafibranor}

Elafibranor (ELA, GFT505) is a dual PPARa/ $\delta$ and was examined in a phase $2 \mathrm{~b}$ trial: GOLDEN 505 (ClinicalTrials.gov ID: NCT01694849). Two different doses (80 and $120 \mathrm{mg}$ ) were tested in 276 patients with biopsy-controlled NASH [17]. In the intention-to-treat analysis, the primary endpoint, defined as the absence of at least one out of three histological components of NASH (steatosis, ballooning, and inflammation) without worsening of fibrosis was not meet [17]. However, a postdoc analysis using the updated definition of NASH resolution (disappearance of ballooning with either disappearance or persistence of mild inflammation) showed that treatment with $120 \mathrm{mg}$ elafibranor resolved NASH in a higher proportion of patients than placebo ( 19 vs. $12 \%, p=0.045$ ) [17]. The reversible and mild increase in serum creatinine appears to be related to the PPAR class. The large, ongoing phase 3 RESOLVE-IT trial will clarify its effects in NASH with moderate and advanced fibrosis.

\section{Metabolic Hormones and Drugs}

One driver of disease progression is steatohepatitis that emerges in metabolic-burdened individuals. Therefore, drugs that exert beneficial metabolic effects have been studied for the treatment of NASH previously. Among these, metformin is frequently used in patients with type 2 diabetes and NASH, but no significant improvement related to the histologically defined endpoints resolution of steatohepatitis or improvement in fibrosis were shown in available studies despite the benefit that metformin helps to control diabetes [18].

The gut-derived hormone glucagon-like peptide-1 (GLP-1) improves insulin secretion, suppresses appetite, and delays gastric emptying. Treatment with GLP-1 analogues ameliorates glycemic control and is also linked to decreased serum ALT levels, suggesting their potential use in NASH treatment. A small phase 2 trial in 52 patients explored the GLP-1 analogue liraglutide (ClinicalTrials.gov ID: NCT01237119). The LEAN study demonstrated a beneficial effect in obese patients with NASH and fibrosis of F0-F4 leading to a superior rate of improvement in steatohepatitis without worsening of fibrosis [19]. NASH resolution, defined as the disappearance of hepatocyte ballooning, was seen in $39 \%$ of patients on liraglutide, and the progression of fibrosis was reduced in $36 \%$ of treated patients. The development of GLP-1 agonists that have a prolonged effect might increase acceptance in patients. In weight loss trials, ALT reduction was observed in patients with elevated ALT at baseline using semaglutide that is injected once weekly. Semaglutide is currently evaluated in phase 2 trials for the indication $\mathrm{NASH}$, and an orally available compound has been approved by the FDA, thus further increasing the potential of this drug in the treatment of NASH.

The class of SGLT-2 inhibitors are promising, because they have been shown to exert beneficial cardiovascular effects [20]. The data available in NASH are very limited. Mostly single-arm, uncontrolled trials have been reported up to date. Empagliflozin showed improvement in steatohepatitis at 24 weeks in a small trial including 9 patients [21]. Based on the strong metabolic effects, these drug types appear promising in combination, and future studies will explore their relative contribution to the improvement in steatohepatitis.

MGL-3196 (resmetirom) is a liver-specific and selective thyroid $\beta$-receptor agonist that was previously explored for its effects on LDL-cholesterol and triglycerides. In a phase 2 trial, patients with biopsy-proven NASH and 
$>10 \%$ liver fat (defined as magnetic resonance imaging, MRI, proton density fat fraction, PDFF, $>10 \%$ ) were treated with MGL-3196 or placebo for 36 weeks. The primary endpoint assessed a number of parameters including liver fat measured by MRI-PDFF at the 12- and 36week follow-ups. A reduction in intrahepatic fat was observed in patients who received either MGL-3196 or placebo with a reduction in MRI-PDFF of $36 \%$ at week 12 and $37 \%$ at week 36 with the lower dose and 42 and $49 \%$ with the higher dose, while placebo achieved 8 and $10 \%$, respectively. Liver enzymes improved more in the active arm, and the dug was generally well tolerated [22]. Based on these positive data, a phase 3 trial was started in the second quarter of 2019 (Table 1).

Aramchol is a synthetic lipid-fusion molecule that combines a bile acid and saturated fatty acids and inhibits stearoyl-CoA-desaturase (SCD)-1. It is through this mechanism that the hepatic triglyceride content is reduced. In the phase $2 \mathrm{~b}$ ARREST study, significant reductions in transaminases, $\mathrm{HbA}_{1 \mathrm{c}}$, and intrahepatic fat deposition were achieved at 52 weeks. While the study did not show significant differences, resolution of NASH at a dose of $2 \times 300 \mathrm{mg}$ without worsening of fibrosis was observed in 16.7 versus $5.0 \%(p=0.051)$ [23]. Importantly, SCD-1 activates AMPK, improves insulin sensitivity, and reduces $\mathrm{HbA}_{1 \mathrm{c}}$. Therefore, the expectations are high that these positive results will read through in the large ARMOR trial - in particular as only few trials so far were able to show a benefit on improved glycemic control on top of hepatic benefits.

Recently, several members of the fibroblast growth factor (FGF) family have been identified as metabolic hormones with beneficial effects on NAFLD [24]. FGF signaling has raised several concerns regarding adverse effects and a short half-life, but the availability of FGF analogues with improved pharmacokinetic and pharmacodynamic profiles has boosted the clinical development of this drug class. The intestinal hormone FGF19 analogue NGM282 (ClinicalTrials.gov ID: NCT02443116) was tested in patients with biopsy-confirmed NASH and resulted in a clinically relevant decrease in hepatic fat content (defined as $\geq 30 \%$ as determined by MRI-PDFF) in $>80 \%$ of patients, in parallel to decreasing transaminases and enhanced liver fibrosis (ELF) scores, suggestive of decreasing fibrosis [25]. In a subset of patients treated with NGM282, improved histological features were reported as early as 12 weeks.

FGF21 is another member of the FGF family and exerts its biological effects by binding to FGF receptors complexed with the transmembrane protein $\beta$ Klotho (KLB). FGF21 is primarily produced by the liver in response to metabolic stress, such as fasting or a ketogenic diet. Increased FGF21 plasma levels are associated with metabolic disease, including obesity, type 2 diabetes, and
NAFLD/NASH [26]. FGF21 administration promotes insulin-sensitizing effects that are linked to the FGFR1-KLB complex in adipose tissue and the CNS [27]. Several FGF21 analogues have been abandoned for short-term effects of safety concerns. However, a recent form that has been pegylated for long-term exposure, pegbelfermin, is now entering late-stage clinical development. In the phase 2a study, 184 overweight or obese NASH patients were treated with 2 doses or placebo. A prespecified interim analysis at 8 weeks showed a significant decrease in the absolute hepatic fat fraction in the group receiving $10 \mathrm{mg}$ pegbelfermin daily $(-6.8 \%, p=0.0004)$ and in the group receiving $20 \mathrm{mg}$ pegbelfermin weekly $(-5.2 \% ; p=0.008)$ compared with the placebo group (-1.3\%). Most adverse events were mild; the most common events were diarrhea in $16 \%$ and nausea in $14 \%$ of patients treated with pegbelfermin [28].

24-Norursodeoxycholic acid (Nor-UDCA) is a synthetic side chain-shortened C23 UDCA homologue that undergoes hepatic metabolism and enriches specifically in hepatocytes. It was found to reduce hepatic steatosis, inflammation, fibrosis, and apoptosis in mouse models of NAFLD or NASH (e.g., by downregulating lipogenic and apoptotic pathways) [29]. In a phase 2 a study of 198 individuals included in the analysis, a dose-dependent reduction in serum ALT levels at baseline versus the last treatment follow-up was observed in the Nor-UDCA group versus the placebo group, with a significant effect in the group treated with $1,500 \mathrm{mg}$ (mean change $-27.8 \%$; $p<$ $0.0001)$. Serious adverse events were comparable to the placebo group [30]. Based on this, a phase $2 \mathrm{~b}$ trial was initiated in Europe (Eudra CT: 2019-003443-31).

\section{Antifibrotic Drugs}

Direct antifibrotic medications are currently not available, but the inhibition of injury - as the driver of fibrosis in liver disease - can lead to fibrosis reversal. Since fibrosis is linked to clinical outcome, including overall mortality, pivotal trials are frequently assessing fibrosis resolution as the primary endpoint. Cenicriviroc (CVC) is a dual inhibitor of the chemokine receptors CCR2 und CCR5, which inhibit intra- and extrahepatic immune cell activation, predominantly macrophages [31]. In the phase $2 \mathrm{~b}$ CENTAUR trial, 289 patients with NASH and fibrosis stages F1-F3 were examined over 24 months. This trial, which used 3 liver biopsies to assess treatment response within 2 years, demonstrated superiority of CVC compared to placebo. While the overall assessment was limited by a number of patients that withdrew consent to undergo the 2-year biopsy, overall the results were promising enough for CVC to be tested in a phase 3 trial. This has recently randomized enough patients to allow for the 
Fig. 1. Pathophysiology of NASH and drugs in late-stage clinical development. The pathophysiology of NASH involves altered metabolism, hepatic inflammation, and fibrogenesis. Visceral obesity, the gutvascular barrier, and hepatic activation of immune cells are centrally involved in the pathogenesis of fibrosis progression in NAFLD. Several drugs are in clinical development and address one or several of these mechanisms. The placement in the figure depicts a primary mechanism of action by which a specific drug is thought to affect NASH - albeit most of the compounds have multiple mechanisms and suppression of inflammation will most likely be beneficial for hepatic fibrosis.

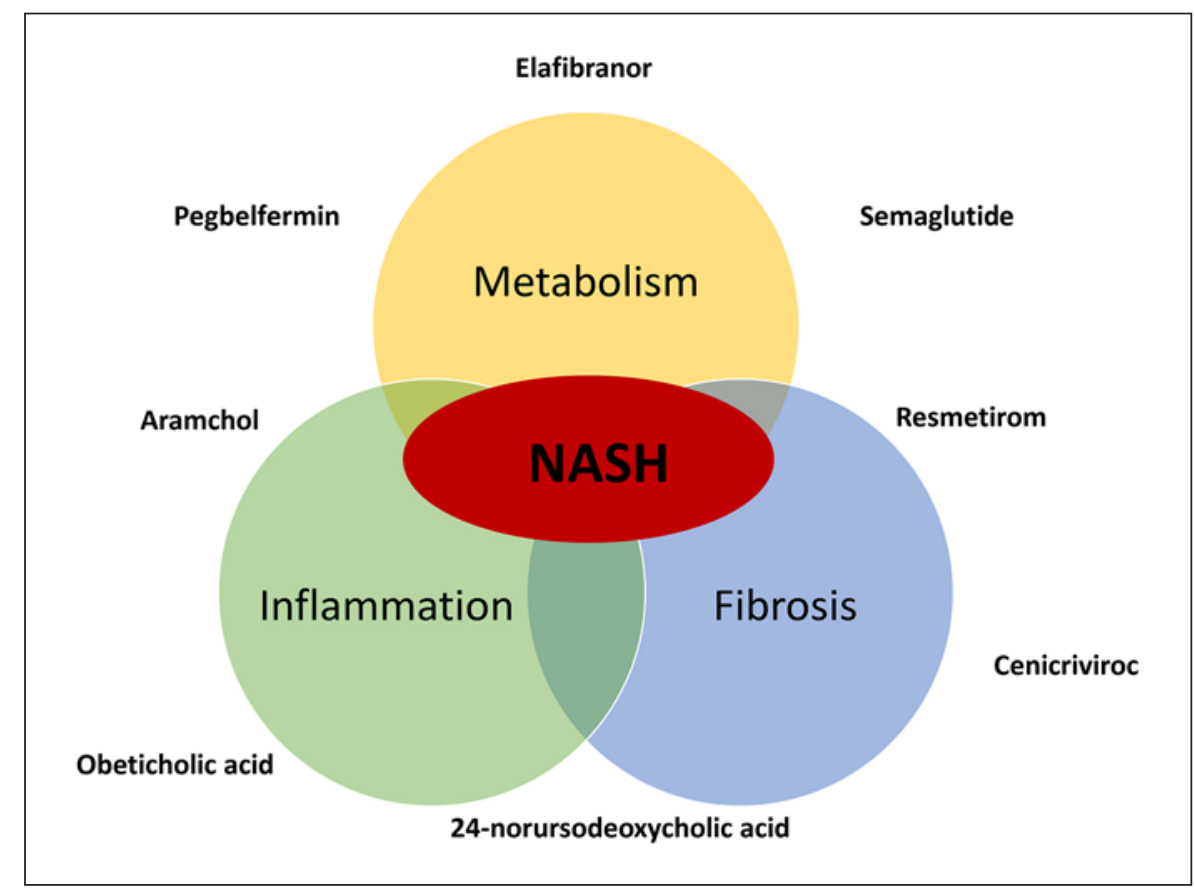

prespecified interim analysis, and results are eagerly awaited this year.

Two drug candidates that were evaluated in trials that enrolled patients with NASH cirrhosis recently reported negative results. Selonsertib, an ASK-1 inhibitor, failed to achieve reversal of cirrhosis and fibrosis in the STELLAR-4 and STELLAR-3 trials, and thus will not be further explored in monotherapy. Likewise, the caspase inhibitor emricasan did not meet the primary endpoint in both a precirrhotic and a cirrhotic patient population [32]. Interestingly, here, fibrosis tended to even worsen in the active treatment arm. Thus, both trials highlight the urgent need of robust preclinical data that needs to be interpreted in light of the models employed before introducing the treatment concept in patients in a clinical trial.

\section{Combination Therapy}

Based on the multimodal pathophysiology of NASH that involves metabolic deterioration, hepatic necroinflammation, and fibrogenesis (Fig. 1), it has been suggested that the inhibition of more pathways could improve the low response rates that are reported from monotherapy. Depending on the type of combination, synergistic mechanisms could lead to the potentiation of the efficacy or improved safety by reducing the required dosage of the individual compounds. At present, the regulatory barriers to combination therapies are higher than for monotherapies because the trials are more complex and larger. Nonetheless, several combinations are explored in phase 2 studies. The TANDEM study combines the FXR agonist tropifexor and CVC in patients with stage F2 and F3 fibrosis. Another combination examines anti-inflammatory, antifibrotic, and metabolic substances using selonsertib (ASK1 inhibition), cilofexor (FXR agonist, GS-9674) and firsocostat (ACC inhibitor, GS-0976) in patients with NASH and advanced fibrosis (ATLAS studies). In a phase 2 study, the GLP-1 agonist semaglutide is currently combined with the above-mentioned cilofexor and firsocostat for the treatment of NASH highlighting that currently multidrug therapy is thought to overcome the limitations of monotherapy in a pathophysiological complex disease.

\section{Disclosure Statement}

J.M.S. declares consultancy (BMS, Boehringer Ingelheim, Echosens, Genfit, Gilead Sciences, Intercept Pharmaceuticals, IQVIA, Madrigal, Novartis, Pfizer, and Roche), research funding (Gilead Sciences), and lecture fees (AbbVie, Falk Foundation, and MSD).

\section{Funding Sources}

J.M.S. is partly funded by the European Union Innovative Medicines Initiative 2 (IMI2) Joint Undertaking under grant agreement 777377: LITMUS (Liver Investigation: Testing Biomarker Utility in Steatohepatitis) consortium. 


\section{References}

1 Dulai PS, Singh S, Patel J, Soni M, Prokop LJ, Younossi Z, et al. Increased risk of mortality by fibrosis stage in nonalcoholic fatty liver disease: systematic review and meta-analysis. Hepatology. 2017 May;65(5):1557-65.

2 Labenz C, Huber Y, Kalliga E, Nagel M, Ruckes C, Straub BK, et al. Predictors of advanced fibrosis in non-cirrhotic non-alcoholic fatty liver disease in Germany. Aliment Pharmacol Ther. 2018 Nov;48(10):1109-16.

3 Wing RR, Bolin P, Brancati FL, Bray GA, Clark JM, Coday M, et al.; Look AHEAD Research Group. Cardiovascular effects of intensive lifestyle intervention in type 2 diabetes. $N$ Engl J Med. 2013 Jul;369(2):145-54.

4 Vilar-Gomez E, Martinez-Perez Y, Calzadilla-Bertot L, Torres-Gonzalez A, Gra-Oramas B, Gonzalez-Fabian L, et al. Weight loss through lifestyle modification significantly reduces features of nonalcoholic steatohepatitis. Gastroenterology. 2015;149 (2):367-78.

5 Hohenester S, Christiansen S, Nagel J, Wimmer R, Artmann R, Denk G, et al. Lifestyle intervention for morbid obesity: effects on liver steatosis, inflammation, and fibrosis. Am J Physiol Gastrointest Liver Physiol. 2018 Sep;315(3):G329-38.

6 Wong VW, Wong GL, Chan RS, Shu SS, Cheung BH, Li LS, et al. Beneficial effects of lifestyle intervention in non-obese patients with non-alcoholic fatty liver disease. J Hepatol. 2018 Dec;69(6):1349-56.

7 Romero-Gómez M, Zelber-Sagi S, Trenell M. Treatment of NAFLD with diet, physical activity and exercise. J Hepatol. 2017 Oct;67(4): 829-46.

8 Gepner Y, Shelef I, Komy O, Cohen N, Schwarzfuchs D, Bril N, et al. The beneficial effects of Mediterranean diet over low-fat diet may be mediated by decreasing hepatic fat content. J Hepatol. 2019 Aug;71(2):379-88.

9 Huber Y, Pfirrmann D, Gebhardt I, Labenz C, Gehrke N, Straub BK, et al. Improvement of non-invasive markers of NAFLD from an individualised, web-based exercise program. Aliment Pharmacol Ther. 2019 Oct;50(8): 930-9.

10 Bower G, Toma T, Harling L, Jiao LR, Efthimiou E, Darzi A, et al. Bariatric surgery and non-alcoholic fatty liver disease: a systematic review of liver biochemistry and histology. Obes Surg. 2015 Dec;25(12):2280-9.

11 Sanyal AJ, Chalasani N, Kowdley KV, McCullough A, Diehl AM, Bass NM, et al.; NASH CRN. Pioglitazone, vitamin E, or placebo for nonalcoholic steatohepatitis. N Engl J Med. 2010 May;362(18):1675-85.

12 Bril F, Biernacki DM, Kalavalapalli S, Lomonaco R, Subbarayan SK, Lai J, et al. Role of vitamin $\mathrm{E}$ for nonalcoholic steatohepatitis in patients with type 2 diabetes: a randomized controlled trial. Diabetes Care. 2019 Aug; 42(8):1481-8.
13 European Association for the Study of the Liver (EASL)European Association for the Study of Diabetes (EASD)European Association for the Study of Obesity (EASO). EASLEASD-EASO Clinical Practice Guidelines for the management of non-alcoholic fatty liver disease. J Hepatol. 2016 Jun;64(6):1388-402.

14 Neuschwander-Tetri BA, Loomba R, Sanyal AJ, Lavine JE, Van Natta ML, Abdelmalek MF, et al.; NASH Clinical Research Network. Farnesoid X nuclear receptor ligand obeticholic acid for non-cirrhotic, non-alcoholic steatohepatitis (FLINT): a multicentre, randomised, placebo-controlled trial. Lancet. 2015 Mar;385(9972):956-65

15 Younossi ZM, Ratziu V, Loomba R, Rinella M, Anstee QM, Goodman Z, et al.; REGENERATE Study Investigators. Obeticholic acid for the treatment of non-alcoholic steatohepatitis: interim analysis from a multicentre, randomised, placebo-controlled phase 3 trial. Lancet. 2019 Dec;394(10215):2184-96.

16 Labenz C, Prochaska JH, Huber Y, Nagel M, Straub BK, Wild P, et al. Cardiovascular risk categories in patients with nonalcoholic fatty liver disease and the role of low-density lipoprotein cholesterol. Hepatol Commun. 2019 Sep;3(11):1472-81.

17 Ratziu V, Harrison SA, Francque S, Bedossa P, Lehert P, Serfaty L, et al. Elafibranor, an agonist of the peroxisome proliferator-activated receptor- $\alpha$ and $-\delta$, induces resolution of nonalcoholic steatohepatitis without fibrosis worsening. Gastroenterology. 2016 May; 150(5):1147-59.e5.

18 Said A, Akhter A. Meta-analysis of randomized controlled trials of pharmacologic agents in non-alcoholic steatohepatitis. Ann Hepatol. 2017 Jul-Aug; 16(4):538-47.

19 Armstrong MJ, Gaunt P, Aithal GP, Barton D, Hull D, Parker R, et al.; LEAN trial team. Liraglutide safety and efficacy in patients with non-alcoholic steatohepatitis (LEAN): a multicentre, double-blind, randomised, placebocontrolled phase 2 study. Lancet. 2016 Feb; 387(10019):679-90.

20 Zinman B, Wanner C, Lachin JM, Fitchett D, Bluhmki E, Hantel S, et al.; EMPA-REG OUTCOME Investigators. Empagliflozin, cardiovascular outcomes, and mortality in type 2 diabetes. N Engl J Med. 2015 Nov; 373(22):2117-28.

21 Lai LL, Vethakkan SR, Nik Mustapha NR, Mahadeva S, Chan WK. Empagliflozin for the treatment of nonalcoholic steatohepatitis in patients with type 2 diabetes mellitus. Dig Dis Sci. 2020 Feb;65(2):623-31.

22 Younossi Z. Emerging research on MGL3196 for the treatment of nonalcoholic steatohepatitis. Gastroenterol Hepatol (N Y). 2019 Jun;15(6):317-9.
23 Ratziu V, Ladron-De-Guevara L, Safadi R, Poordad F, Fuster F, Harrison S, et al: ARREST investigator study group. One-year results of the global phase $2 \mathrm{~b}$ randomized placebo-controlled arrest trial of aramchol, a stearoyl CoA desaturase inhibitor, in patients with NASH. AASLD Liver Meeting, Abstract. 2018;LB-5:2018.

24 Struik D, Dommerholt MB, Jonker JW. Fibroblast growth factors in control of lipid metabolism: from biological function to clinical application. Curr Opin Lipidol. 2019 Jun;30(3): 235-43.

25 Harrison SA, Rinella ME, Abdelmalek MF, Trotter JF, Paredes AH, Arnold HL, et al. NGM282 for treatment of non-alcoholic steatohepatitis: a multicentre, randomised, double-blind, placebo-controlled, phase 2 trial. Lancet. 2018 Mar;391(10126):1174-85.

26 Dushay J, Chui PC, Gopalakrishnan GS, Varela-Rey M, Crawley M, Fisher FM, et al. Increased fibroblast growth factor 21 in obesity and nonalcoholic fatty liver disease. Gastroenterology. 2010 Aug;139(2):456-63.

27 Lan T, Morgan DA, Rahmouni K, et al. FGF19, FGF21, and an FGFR1/beta-Klothoactivating antibody act on the nervous system to regulate body weight and glycemia. Cell Metab. 2017;26(5):709-18.e3.

28 Sanyal A, Charles ED, Neuschwander-Tetri BA, Loomba R, Harrison SA, Abdelmalek MF, et al. Pegbelfermin (BMS-986036), a PEGylated fibroblast growth factor 21 analogue, in patients with non-alcoholic steatohepatitis: a randomised, double-blind, placebo-controlled, phase 2a trial. Lancet. 2019 Dec;392(10165):2705-17.

29 Beraza N, Ofner-Ziegenfuss L, Ehedego H, Boekschoten M, Bischoff SC, Mueller M, et al. Nor-ursodeoxycholic acid reverses hepatocyte-specific nemo-dependent steatohepatitis. Gut. 2011 Mar;60(3):387-96.

30 Traussnigg S, Schattenberg JM, Demir M, Wiegand J, Geier A, Teuber G, et al.; Austrian/German NAFLD-norUDCA study group. Norursodeoxycholic acid versus placebo in the treatment of non-alcoholic fatty liver disease: a double-blind, randomised, placebocontrolled, phase 2 dose-finding trial. Lancet Gastroenterol Hepatol. 2019 Oct;4(10):78193.

31 Friedman S, Sanyal A, Goodman Z, Lefebvre E, Gottwald M, Fischer L, et al. Efficacy and safety study of cenicriviroc for the treatment of non-alcoholic steatohepatitis in adult subjects with liver fibrosis: CENTAUR Phase $2 \mathrm{~b}$ study design. Contemp Clin Trials. 2016 Mar; 47:356-65.

32 Harrison SA, Goodman Z, Jabbar A, Vemulapalli R, Younes ZH, Freilich B, et al. A randomized, placebo-controlled trial of emricasan in patients with NASH and F1-F3 fibrosis. J Hepatol. 2020 May;72(5):816-827. 